\title{
Histological Findings of Bone Marrow Lesions on Magnetic Resonance Images in Patients with Varus Knee Osteoarthritis
}

\author{
Takehiko Sugita1*, Tomomaro Kawamata², Toshimi Aizawa ${ }^{3}$, Akira Sasaki1, \\ Naohisa Miyatake ${ }^{1}$, Takeshi Uehara ${ }^{1}$ \\ ${ }^{1}$ Department of Orthopaedic Surgery, Tohoku Orthopaedic Clinic, Sendai, Japan \\ ${ }^{2}$ Department of Orthopaedic Surgery, Sendai Orthopaedic Hospital, Sendai, Japan \\ ${ }^{3}$ Department of Orthopaedic Surgery, Tohoku University Graduate School of Medicine, Sendai, Japan \\ Email: ${ }^{*}$-sugita@tohokuseikei.com
}

Received 27 September 2014; revised 12 November 2014; accepted 28 November 2014

Copyright (C) 2014 by authors and Scientific Research Publishing Inc.

This work is licensed under the Creative Commons Attribution International License (CC BY).

http://creativecommons.org/licenses/by/4.0/

(c) (i) Open Access

\begin{abstract}
Bone marrow lesions (BMLs) on magnetic resonance (MR) images in knee osteoarthritis patients are considered to predict the severity and progression of the disease. We evaluated the histological findings of BMLs on MR images of the subchondral area of the medial femoral condyle in varus osteoarthritic knees. In 24 patients with varus knee osteoarthritis who underwent total knee arthroplasty (TKA), sagittal T1- and T2-weighted MR images of the affected knee were acquired before TKA. During TKA, resected bone pieces from the distal medial femoral condyle were obtained. Sagittal specimens obtained from the center of the bone pieces were histologically examined. Twenty patients had BMLs. Histological findings of BMLs in the subchondral area showed various features, such as fibrovascular tissue, cyst formation, active bone remodeling with bone formation and bone resorption, and hyaline cartilage. BMLs were not found in four patients; histological findings of these patients showed normal bone marrow tissue with normal-thickness trabeculae. Subchondral bony end plate in knees with BMLs was usually thin or destroyed, while that without BMLs was thick or normal. The condition of the subchondral bony end plate would explain the differences in the severity and progression between patients with or without BMLs.
\end{abstract}

\section{Keywords}

Knee Osteoarthritis, Magnetic Resonance Images, Histology, Bone Marrow Lesion

\footnotetext{
${ }^{*}$ Corresponding author.
}

How to cite this paper: Sugita, T., Kawamata, T., Aizawa, T., Sasaki, A., Miyatake, N. and Uehara, T. (2014) Histological Findings of Bone Marrow Lesions on Magnetic Resonance Images in Patients with Varus Knee Osteoarthritis. Open Journal of Orthopedics, 4, 327-334. http://dx.doi.org/10.4236/ojo.2014.412050 


\section{Introduction}

Osteoarthritis of the knee is extremely common. Nearly $75 \%$ of individuals aged $\geq 65$ years in the United States have evidence of degenerative joint disease on knee radiographs [1]. A recent Japanese epidemiological study revealed that the prevalence of radiographic knee osteoarthritis was $42.6 \%$ in men and $62.4 \%$ in women aged $\geq 40$ years [2]. Because knee osteoarthritis reduces the quality of life of elderly people, the high incidence of osteoarthritis and increased average lifespan have resulted in worldwide social problems in terms of reduced mobility. To address these issues, prediction and prevention —in addition to treatment of osteoarthritis-have become increasingly important.

In order to predict the severity and progression of osteoarthritis, several methods such as biological markers [3] and diagnostic imaging [4]-[6] have been used to date. Bone marrow lesions (BMLs) on magnetic resonance (MR) images, which have been previously defined as "ill-defined low-signal-intensity areas on T1-weighted images (T1WI)" [7] and "high-signal-intensity areas on T2-weighted images (T2WI)" [8] [9] within subchondral regions, have been reported to correlate with the severity [8] [10], progression [11] [12] and cartilage damage [13]-[16] of knee osteoarthritis. However, the exact pathology of BMLs remains uncertain since only a few studies have investigated the histological findings of these lesions [7] [17] [18] and no histological explanations of the differences in the severity and progression between patients with or without BMLs have been reported. Therefore in the present study, we evaluated histological findings of the subchondral area of the medial femoral condyle with BMLs on MR images and compared them with those without BMLs.

\section{Patients and Methods}

The study protocol was approved by the Ethics Committee of our clinic. Written informed consent for the use of data in the study was obtained from all patients.

\subsection{Patients}

Between October 2012 and February 2013, 24 total knee arthroplasties (TKAs) were consecutively performed for 24 patients with varus knee osteoarthritis. Those patients were the subjects of this study. The study comprised 4 males and 20 females. The demographic data of the patients are shown in Table 1 . The average age at surgery, height, weight and body mass index were 72 years (range 62 - 80 years), $1.52 \mathrm{~m}$ (range 1.39 - $1.75 \mathrm{~m}$ ), $66 \mathrm{~kg}$ (range 54 - $89 \mathrm{~kg}$ ) and $28.3 \mathrm{~kg} / \mathrm{m}^{2}$ (range $23.3-37.2 \mathrm{~kg} / \mathrm{m}^{2}$ ), respectively. Grade of osteoarthritis in all patients showed grade 4 according to Kellgren and Lawrence grading system [19]. The average femoro-tibial angle on a standing posteroanterior radiograph was $186^{\circ}$ (range $\left.179^{\circ}-198^{\circ}\right)$.

\subsection{MRI Examinations}

MR images of the affected knee were acquired before the TKA. In our clinic, a Signa-Profile system (0.2 T; GE Healthcare, Milwaukee, WI) is used routinely to acquire T1- and T2-weighted sagittal and coronal images to assess knee pathologies. The section thickness of the sagittal planes was $4 \mathrm{~mm}$. Because the patients had varus knee osteoarthritis, we used T1- and T2-weighted sagittal MR images through the center of the medial femoral condyle for the imaging evaluation of the patients. BMLs were generally represented by ill-defined areas of low-signal intensity on T1WI [7] and by areas of high-signal intensity on T2WI [8] [9] in the subchondral area (Figure 1).

\subsection{Histological Examinations}

During the TKA, bone pieces were obtained from the distal medial femoral condyle. They were cut sagittally and the specimens-approximately $5 \mathrm{~mm}$ thick, from the center of the medial femoral condyle-were prepared for histological examinations (Figure 2). The specimens were immediately fixed in $10 \%$ formalin, decalcified with ethylenediaminetetraacetic acid at $4{ }^{\circ} \mathrm{C}$, embedded in paraffin, and stained with hematoxylin-eosin and elastica-Masson. Antibodies against type-II collagen were also used to prepare the specimens for immunohistological examination. Stained specimens were analyzed using light microscopy.

\section{Results}

The MR images revealed BMLs for 20 patients but no BMLs for 4 patients. All the lesions were confirmed by 
Table 1. Demographic data of the patients in the present study.

\begin{tabular}{|c|c|c|c|c|c|c|c|}
\hline No. & Age & Sex & $\mathrm{R} / \mathrm{L}$ & Height (m) & Weight (kg) & $\operatorname{BMI}\left(\mathrm{kg} / \mathrm{m}^{2}\right)$ & Femoro-Tibial Angle \\
\hline 1 & 66 & $\mathrm{~F}$ & $\mathrm{R}$ & 1.54 & 71 & 29.9 & 184 \\
\hline 2 & 69 & $\mathrm{~F}$ & $\mathrm{~L}$ & 1.5 & 67 & 29.7 & 181 \\
\hline 3 & 62 & $\mathrm{~F}$ & $\mathrm{~L}$ & 1.47 & 68 & 31.4 & 184 \\
\hline 4 & 76 & $\mathrm{~F}$ & $\mathrm{~L}$ & 1.5 & 62 & 27.5 & 185 \\
\hline 5 & 79 & $\mathrm{~F}$ & $\mathrm{~L}$ & 1.46 & 57 & 26.7 & 198 \\
\hline 6 & 77 & $\mathrm{~F}$ & $\mathrm{~L}$ & 1.48 & 60 & 27.3 & 187 \\
\hline 7 & 78 & $\mathrm{~F}$ & $\mathrm{R}$ & 1.52 & 86 & 37.2 & 179 \\
\hline 8 & 63 & M & $\mathrm{L}$ & 1.71 & 89 & 30.4 & 186 \\
\hline 9 & 73 & $\mathrm{~F}$ & $\mathrm{~L}$ & 1.52 & 59 & 25.5 & 185 \\
\hline 10 & 63 & $\mathrm{~F}$ & $\mathrm{~L}$ & 1.56 & 64 & 26.2 & 180 \\
\hline 11 & 79 & $\mathrm{~F}$ & $\mathrm{~L}$ & 1.51 & 54 & 23.6 & 182 \\
\hline 12 & 64 & $\mathrm{~F}$ & $\mathrm{R}$ & 1.39 & 54 & 27.9 & 191 \\
\hline 13 & 69 & $\mathrm{~F}$ & $\mathrm{R}$ & 1.5 & 58 & 25.7 & 187 \\
\hline 14 & 74 & $\mathrm{M}$ & $\mathrm{R}$ & 1.62 & 68 & 25.9 & 179 \\
\hline 15 & 64 & $\mathrm{M}$ & $\mathrm{L}$ & 1.75 & 89 & 29 & 185 \\
\hline 16 & 79 & $\mathrm{~F}$ & $\mathrm{~L}$ & 1.46 & 63 & 29.5 & 180 \\
\hline 17 & 77 & F & $\mathrm{L}$ & 1.44 & 58 & 27.9 & 182 \\
\hline 18 & 79 & $\mathrm{~F}$ & $\mathrm{~L}$ & 1.59 & 59 & 23.3 & 195 \\
\hline 19 & 71 & $\mathrm{~F}$ & $\mathrm{R}$ & 1.42 & 60 & 29.7 & 185 \\
\hline 20 & 70 & $\mathrm{~F}$ & $\mathrm{~L}$ & 1.56 & 87 & 35.7 & 191 \\
\hline 21 & 76 & $\mathrm{M}$ & $\mathrm{L}$ & 1.61 & 62 & 23.9 & 192 \\
\hline 22 & 76 & $\mathrm{~F}$ & $\mathrm{~L}$ & 1.46 & 57 & 26.7 & 190 \\
\hline 23 & 68 & $\mathrm{~F}$ & $\mathrm{~L}$ & 1.46 & 58 & 27.2 & 190 \\
\hline 24 & 80 & $\mathrm{~F}$ & $\mathrm{R}$ & 1.43 & 54 & 26.4 & 186 \\
\hline
\end{tabular}

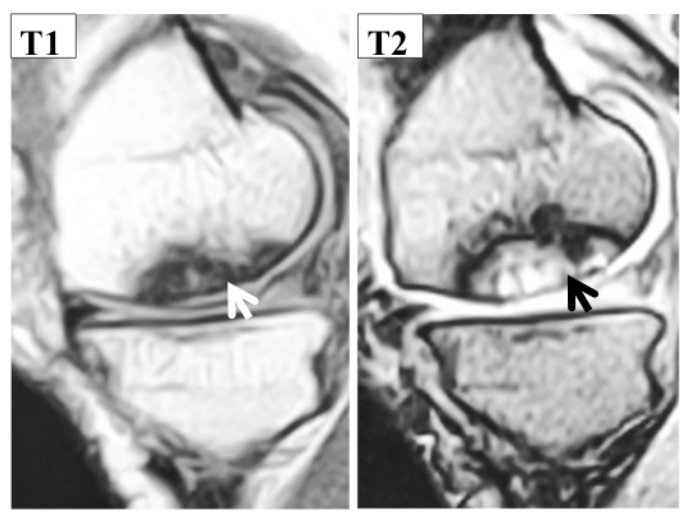

Figure 1. T1- and T2-weighted MR images of bone marrow lesions. Diffuse low-signal intensity on T1-weighted images (T1, white arrow) and high-signal intensity on T2-weighted images (T2, black arrow) demonstrates bone marrow lesions.

imaging of the middle slice of the medial femoral condyle. Plain radiographs showed no differences between patients with or without BMLs on MR images (Figure 3(a), Figure 3(b)). Histologically, BMLs were shown to exhibit various features: the formation of fibrofatty or fibrovascular bone marrow; cyst formation (Figure 4(a)); active bone remodeling with bone formation and resorption (Figure 4(b)); and the formation of cartilaginous tissues, including articular cartilage, subchondral bony end plate and bone trabeculae. In all 20 patients, the articular cartilage either partially remained or had completely disappeared. Bared subchondral bony end plate was thin or disintegrated. Dense fibrous or fibrovascular bone marrow tissues with thick trabeculae were observed 


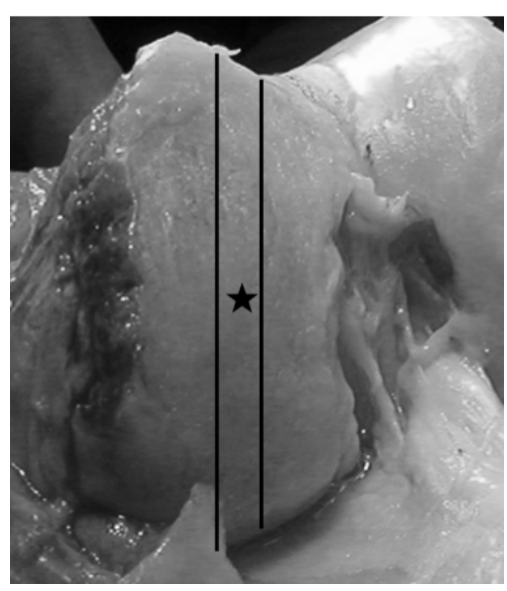

Figure 2. Photo of medial femoral condyle during total knee arthroplasty. A central sagittal specimen (asterisk) was obtained from the resected distal medial femoral condyle.

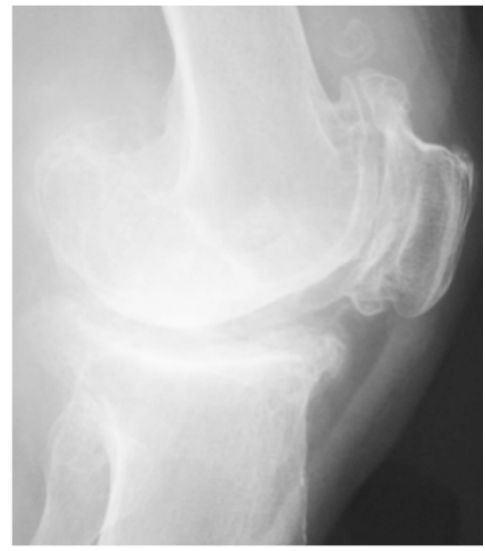

(a)

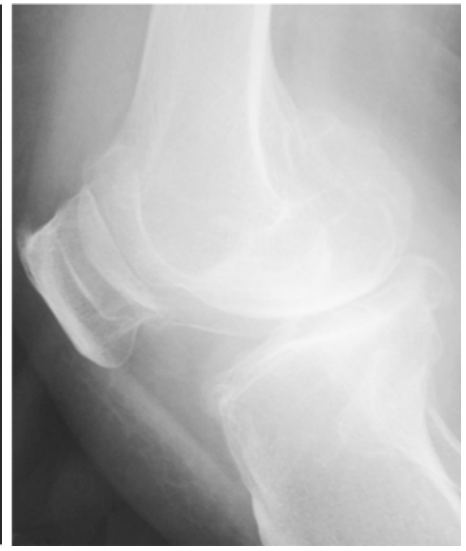

(b)

Figure 3. Plain radiographs of the patients with or without BMLs on MR images. No obvious differences can be detected by the plain radiographs. (a) A plain radiograph of the patient with BMLs; (b) A plain radiograph of the patient without BMLs.

under the end plate (Figure 4(c)). Bone necrosis with empty lacunae was occasionally found in this end plate. After immunostaining for type-II collagen, cartilaginous tissues were detected not only on the surface of the specimen but also in the subchondral region (Figure 4(d)). In some specimens, articular cartilage partially remained. Moderately fibrofatty or fibrovascular bone marrow and normal-thickness trabeculae were observed beneath a thin or disintegrated subchondral bony end plate (Figure 4(e)).

The histological findings of the four patients with no BMLs showed normal bone marrow tissue beneath either well-preserved articular cartilage with a normal-thickness subchondral bony end plate (Figure 5(a)) or a completely bared articular surface with a thick subchondral bony end plate (Figure 5(b)). Cyst formation or bone necrosis were not found.

\section{Discussion}

BMLs are defined, based on MRI findings, as "low-signal-intensity on T1WI” [7] and "high-signal-intensity on T2WI” [8] [9] within subchondral regions. Recently, many studies have revealed an association between BMLs and knee pain and the structural progression of osteoarthritis [8]-[16]. Felson et al. [8] reported that BMLs were strongly associated with the presence of pain. Iwasaki et al. [10] stated that irregularity of BMLs served as a possible indicator of objective severity when assessed by the Lysholm knee scoring scale. A couple of longitu- 


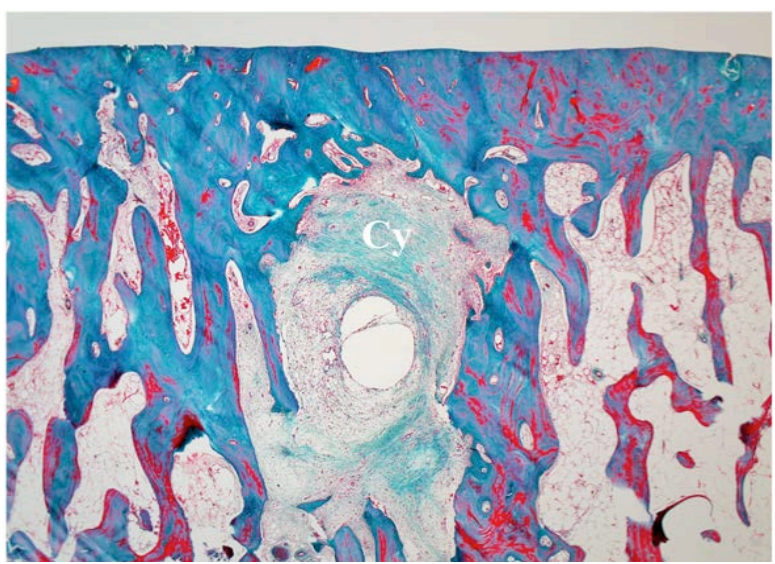

(a)

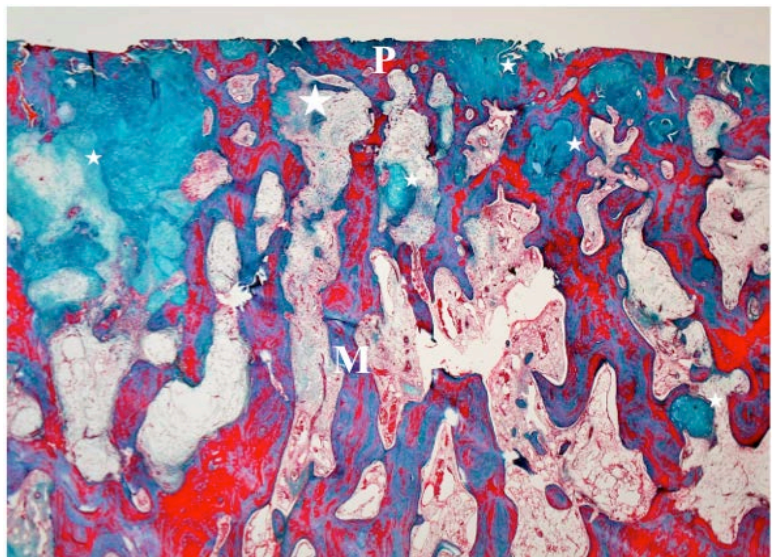

(c)

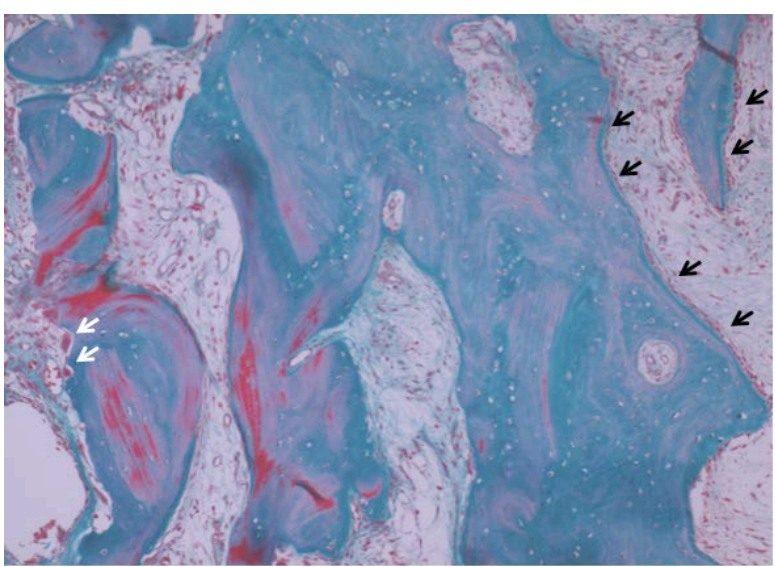

(b)

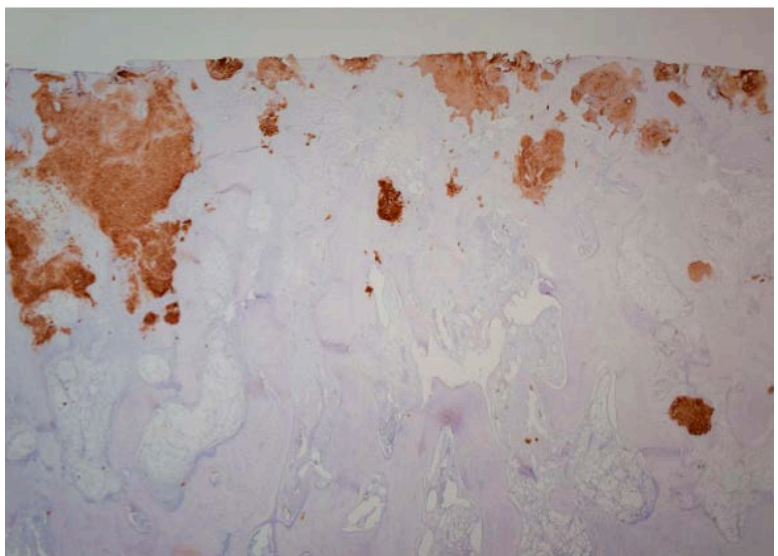

(d)

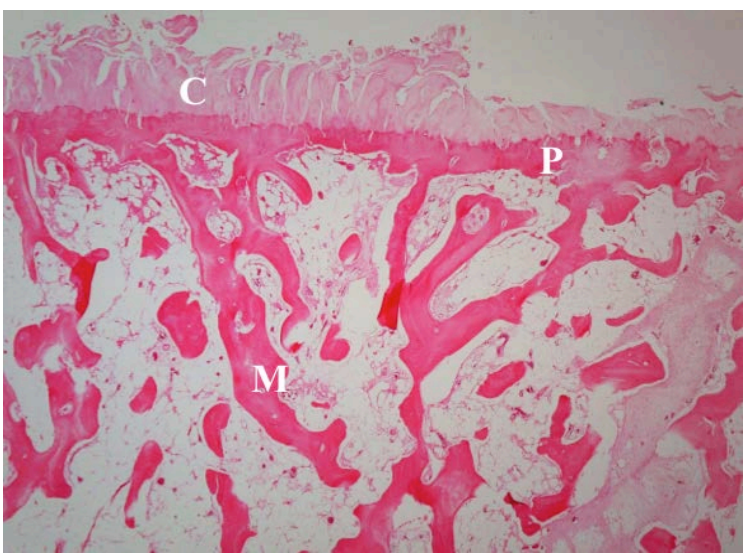

(e)

Figure 4. Histological features of bone marrow lesions. (a) Stained with elastica-Masson, original magnification $\times 20$; (b) Stained with elastica-Masson, original magnification $\times 100$; (c) Stained with elastica-Masson, original magnification $\times 20$; (d) Stained with antibodies against type-II collagen, original magnification $\times 20$; (e) Stained with hematoxylin-eosin, original magnification $\times 20$. Cy: cyst formation; C: articular cartilage; M: bone marrow region; P: subchondral bony end plate. (a) Cyst formation is observed in the bone marrow lesion; (b) Active bone remodeling with bone formation (black arrow) and resorption (white arrow) are observed; (c) Thick trabeculae and dense fibrofatty or fibrovascular marrow tissues are observed under bared and thin or disintegrated subchondral bony end plate. Cartilaginous tissue (asterisk) is also observed; (d) The cartilaginous tissue shows positive staining for type-II collagen; (e) Normal-thickness trabeculae and moderately fibrofatty or fibrovascular marrow are observed under partially remaining articular cartilage and thin or disintegrated subchondral bony end plate. 


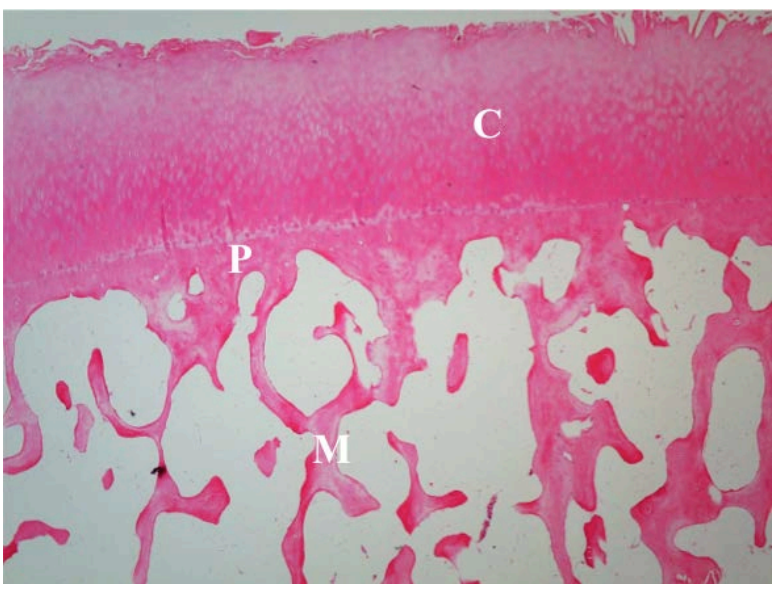

(a)

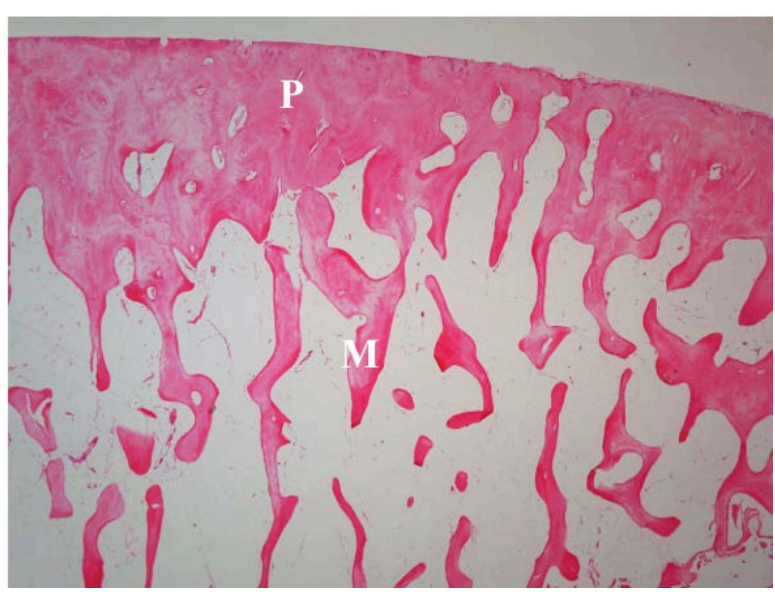

(b)

Figure 5. Histological features of the knees with no bone marrow lesions (stained with hematoxylin-eosin, original magnification 20×). (a) Well-preserved articular cartilage, normal-thickness subchondral bony end plate, normal-thickness trabeculae, and normal bone marrow tissue are observed; (b) Bared subchondral bony end plate, thick subchondral bony end plate, normal-thickness trabeculae, and normal bone marrow tissue are observed. (C: articular cartilage; M: bone marrow region; P: subchondral bony end plate).

dinal studies revealed a significant relationship between BMLs and progression of knee osteoarthritis [11] [12]. Studies have also shown an association between BMLs and articular cartilage damage [13]-[16]. Lim et al. [9] reviewed the association between non-biomechanical factors and BMLs. Therefore, BMLs should play an important role in the etiology of osteoarthritis; however, only a few studies have been conducted in which the histology of BMLs has been directly observed [7] [17] [18].

Zanetti et al. [7] compared tibial plateau abnormalities on MR images with those on histological maps for 16 patients. They concluded that the bone marrow edema depicted on MR images in osteoarthritis knees mainly consisted of normal tissue with smaller areas of various abnormalities, such as bone marrow necrosis, necrotic or remodeled trabeculae, bone marrow fibrosis, bone marrow edema and bone marrow bleeding. In another study, Bergman et al. [17] reported that seven of nine osteoarthritis knees exhibited MR signal abnormalities corresponding predominantly to the replacement of fatty marrow with fibrous tissue. In addition, Sadat et al. [18] observed subchondral ingrowth of fibrovascular tissue and increased bone remodeling in the bone marrow edema pattern zone on the MR images of one patient. Although such studies have explored an aspect of BML pathology, the full and exact significance of the lesions is yet to be elucidated.

In the present study, the center of the medial femoral condyle-where BMLs were confirmed by MR imaging - was histologically examined. In addition, histological differences between patients with or without BMLs were evaluated. BMLs were shown to exhibit various histological features: the formation of fibrofatty or fibrovascular bone marrow; cyst formation; active bone remodeling with bone formation and resorption; and the hyaline cartilage in the subchondral region. Nevertheless, the MR images of the four patients with no BMLs revealed normal bone marrow tissues. The most interesting histological difference between patients with or without BMLs was the condition of the subchondral bony end plate; the end plate was thin or disintegrated in patients with BMLs but thick in patients with no BMLs. Although histological findings of BMLs in this study were similar to those of previous studies [7] [17] [18], those studies did not describe the condition of the subchondral bony end plate in detail, regrettably. Based on the current study, the condition of the subchondral bony end plate should play a key role in the pathology of BMLs and should explain the differences in the severity and progression of knee osteoarthritis between patients with or without BMLs. It is not known whether the disintegration of the subchondral bony end plate is the cause or result of the underlying pathology of BMLs. However, once the plate has been disintegrated, the bone marrow beneath the plate becomes directly exposed by compression force, which may accelerate the disease progression of osteoarthritis. Furthermore, once the plate is disintegrated, unmyelinated free nerve endings present in the subchondral bone can presumably worsen the severity of osteoarthritic pain [20].

In addition to the relatively small sample size and imbalance in the number of samples with or without BMLs, 
the current study has some limitations. First, only 0.2 T T1- and T2-weighted MR images, which were routinely acquired in our clinical practice, could be used for this study. Such MR images cannot detect cartilage, but can identify BMLs. Second, the specimens examined in this study were obtained from advanced osteoarthritis knees during TKAs. Therefore, it may be difficult to surmise the etiology of BMLs in early-stage osteoarthritis knees and the progression of the lesions. Finally, it was very difficult to determine the exact correlations between the BMLs and the histological findings since the MR images were acquired using sections $3-4 \mathrm{~mm}$ in thickness while histological examinations were conducted on very thin specimens.

\section{Conclusion}

BMLs on MR images of the subchondral area of the medial femoral condyle of varus osteoarthritis knees exhibited various histological findings. The condition of the subchondral bony end plate is likely to influence the histological features of the subchondral area-whether or not BMLs are present. We consider that the histological findings of BMLs are critical to better understand the severity or progression of knee osteoarthritis.

\section{References}

[1] Lawrence, R.C., Hochberg, M.C., Kelsey, J.L., McDuffie, F.C., Medsger, T.A., Felts, W.R. and Shulman, L.E. (1989) Estimates of the Prevalence of Selected Arthritic and Musculoskeletal Disease in the United States. The Journal of Rheumatology, 16, 427-431.

[2] Yoshimura, N., Muraki, S., Oka, H., Mabuchi, A., En-Yo, Y., Yoshida, M., Saika, A., Yoshia, H., Suzuki, T., Yamamoto, S., Ishibashi, H., Kawaguchi, H., Nakamura, K. and Akune, T. (2009) Prevalence of Knee Osteoarthritis, Lumbar Spondylosis, and Osteoporosis in Japanese Men and Women: The Research on Osteoarthritis/Osteoporosis against Disability Study. Journal of Bone and Mineral Metabolism, 27, 620-628. http://dx.doi.org/10.1007/s00774-009-0080-8

[3] Rousseau, J.Ch. and Garnero, P. (2003) Biological Markers in Osteoarthritis. Bone, 51, 265-277. http://dx.doi.org/10.1016/j.bone.2012.04.001

[4] Link, T.M., Steinbach, L.S., Ghosh, S., Ries, M., Lu, Y., Lane, N. and Majumdar, S. (2003) Osteoarthritis: MR Imaging Findings in Different Stages of Disease and Correlation with Clinical Findings. Radiology, 226, 373-381. http://dx.doi.org/10.1148/radiol.2262012190

[5] Akamatsu, Y., Mitsugi, N., Taki, N., Kobayashi, H. and Saito, T. (2012) Medial versus Lateral Condyle Bone Mineral Density Ratio in a Cross-Sectional Study-A Potential Marker for Medial Knee Osteoarthritis Severity. Arthritis Care \& Research, 64, 1036-1045.

[6] Lo, G.H., Tassinari, A.M., Driban, J.B., Price, L.L., Schneider, E., Majumdar, S. and McAlindon, T.E. (2012) CrossSectional DXA and MR Measures of Tibial Periarticular Bone Associate with Radiographic Knee Osteoarthritis Severity. Osteoarthritis and Cartilage, 20, 686-693. http://dx.doi.org/10.1016/j.joca.2012.03.006

[7] Zanetti, M., Bruder, E., Romero, J. and Hodler, J. (2000) Bone Marrow Edema Pattern in Osteoarthritic Knees: Correlation between MR Imaging and Histologic Findings. Radiology, 215, 835-840. http://dx.doi.org/10.1148/radiology.215.3.r00jn05835

[8] Felson, D.T., Chaisson, C.E., Hill, C.L., Totterman, S.M.S., Gale, M.E., Skinner, K.M., Kazis, L. and Gale, D.R. (2001) The Association of Bone Marrow Lesions with Pain in Knee Osteoarthritis. Annals of Internal Medicine, 134, 541-549. http://dx.doi.org/10.7326/0003-4819-134-7-200104030-00007

[9] Lim, Y.Z., Wang, Y., Wluka, A.E., Davies-Tuck, M.L., Biomed, B., Hanna, F., Urquhart, D.M. and Cicuttini, F.M. (2014) Association of Obesity and Systemic Factors with Bone Marrow Lesions at the Knee-A Systematic Review. Seminars in Arthritis and Rheumatism, 43, 600-612. http://dx.doi.org/10.1016/j.semarthrit.2013.10.006

[10] Iwasaki, J., Sasho, T., Nakagawa, K., Ogino, S., Ochiai, N. and Moriya, H. (2007) Irregularity of Medial Femoral Condyle on MR Imaging Serves as a Possible Indicator of Objective Severity of Medial-Type Osteoarthritic Knee-A Pilot Study. Clinical Rheumatology, 26, 1705-1708. http://dx.doi.org/10.1007/s10067-007-0578-1

[11] Felson, D.T., McLaughlin, S., Goggings, J., LaValley, M.P., Gale, M.E., Totterman, S., Li, W., Hill, C. and Gale, D. (2003) Bone Marrow Edema and Its Relation to Progression of Knee Osteoarthritis. Annals of Internal Medicine, 139, 330-337. http://dx.doi.org/10.7326/0003-4819-139-5_Part_1-200309020-00008

[12] Kubota, M., Ishijima, M., Kurosawa, H., Liu, L., Ikeda, H., Osawa, A., Takazawa, Y., Kawasaki, T., Saita, Y., Kimura, Y. and Kaneko, K. (2010) A Longitudinal Study of the Relationship between the Status of Bone Marrow Abnormalities and Progression of Knee Osteoarthritis. Journal of Orthopaedic Science, 15, 641-646. http://dx.doi.org/10.1007/s00776-010-1512-y

[13] Hunter, D.J., Zhang, Y., Niu, J., Goggins, J., Amin, S., LaValley, M.P., Guermazi, A., Genant, H., Gale, D. and Felson, D.T. (2006) Increase in Bone Marrow Lesions Associated with Cartilage Loss. Arthritis and Rheumatism, 54, 1529- 
1535. http://dx.doi.org/10.1002/art.21789

[14] Kijowski, R., Stanton, P., Fine, J. and De Smet, A. (2006) Subchondral Bone Marrow Edema in Patients with Degeneration of the Articular Cartilage of the Knee Joint. Radiology, 238, 943-949. http://dx.doi.org/10.1148/radiol.2382050122

[15] Driban, J.B., Lo, G.H., Lee, J.Y., Ward, R.J., Miller, E., Pang, J., Price, L.L. and McAlindon, T.E. (2011) Quantitative Bone Marrow Lesion Size in Osteoarthritic Knees Correlates with Cartilage Damage and Predicts Longitudinal Cartilage Loss. BMC Musculoskeletal Disorders, 12, 217. http://dx.doi.org/10.1186/1471-2474-12-217

[16] Crema, M.D., Felson, D.T., Roemer, F.W., Wang, K., Marra, M.D., Nevitt, M.C., Lynch, J.A., Torner, J., Lewis, C.E. and Guermazi, A. (2013) Prevalent Cartilage Damage and Cartilage Loss over Time Are Associated with Incident Bone Marrow Lesions in the Tibiofemoral Compartments-The MOST Study. Osteoarthritis and Cartilage, 21, 306313. http://dx.doi.org/10.1016/j.joca.2012.11.005

[17] Bergman, A.G., Willen, H.K., Linddstrand, A.L. and Pettersson, H.T.A. (1994) Osteoarthritis of the Knee-Correlation of Subchondral MR Signal Abnormalities with Histopathologic and Radiographic Features. Skeletal Radiology, 23, 445-448.

[18] Saadat, E., Jobke, B., Chu, B., Lu, Y., Cheng, J., Li, X., Ries, M.D., Majumdar, S. and Link, T.M. (2008) Diagnostic Performance of in Vivo 3-T MRI for Articular Cartilage Abnormalities in Human Osteoarthritic Knees Using Histology as Standard of Reference. European Radiology, 18, 2292-2302. http://dx.doi.org/10.1007/s00330-008-0989-7

[19] Kellgren, J.H. and Lawrence, J.S. (1959) Radiological Assessment of Osteo-Arthrosis. Annals of the Rheumatic Diseases, 16, 494-502. http://dx.doi.org/10.1136/ard.16.4.494

[20] Burr, D.B. (2003) Subchondral Bone in the Pathogenesis of Osteoarthritis. Mechanical Aspect. In: Brandt, K.D., Doherty, M. and Lohmander, L.S., Eds., Osteoarthritis, 2nd Edition, Oxford University Press, Oxford, 161-166. 
Scientific Research Publishing (SCIRP) is one of the largest Open Access journal publishers. It is currently publishing more than 200 open access, online, peer-reviewed journals covering a wide range of academic disciplines. SCIRP serves the worldwide academic communities and contributes to the progress and application of science with its publication.

Other selected journals from SCIRP are listed as below. Submit your manuscript to us via either submit@scirp.org or Online Submission Portal.
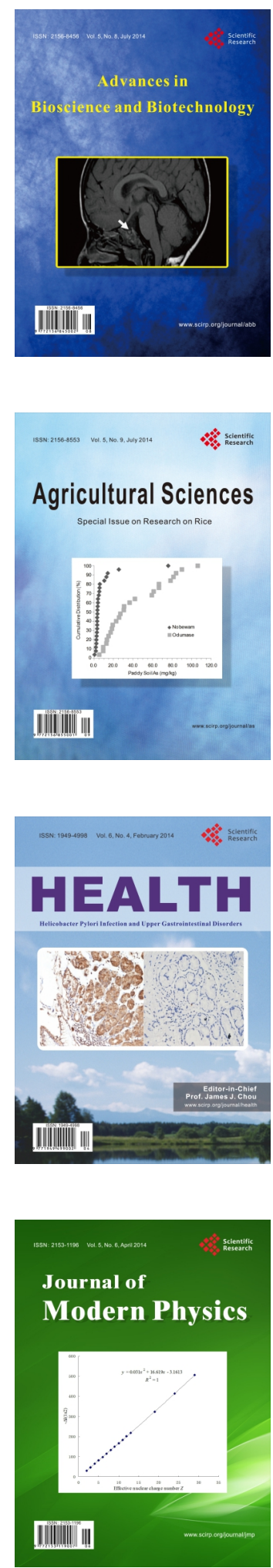
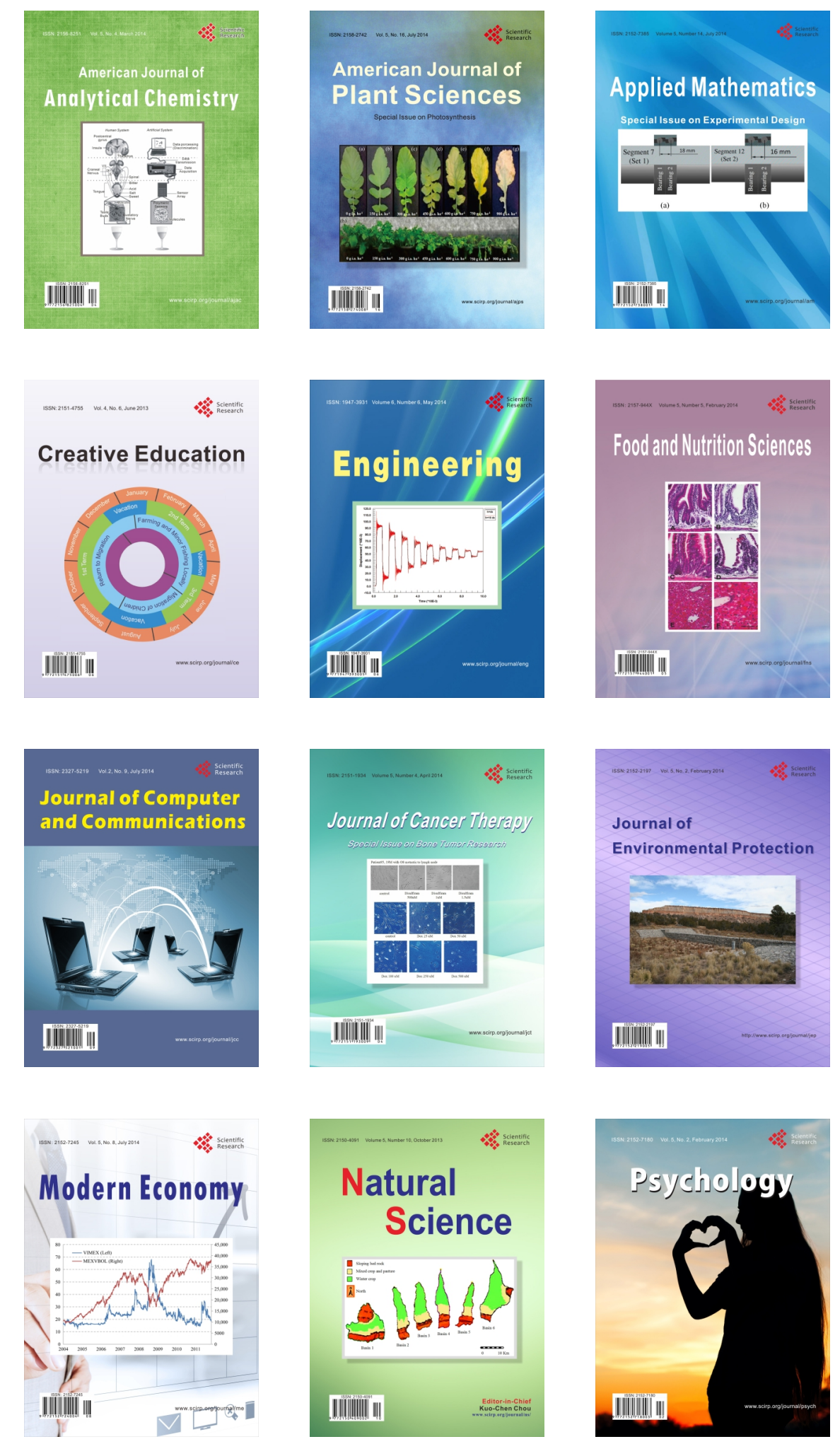